\title{
Tandem-X satellite data application to four- dimensional of copper mineralization
}

\begin{abstract}
Geological mining detections and identifications in synthetic aperture radar (SAR) images are required standard procedures and accurate algorithms. In fact, the main disadvantage of SAR images is speckle noises. The speckles do not allow any accurate retrieving information from SAR data. The main objective of this work is to design an intelligent system based on Particle Swarm Optimization to detect automatically the four-dimensional of copper mineralization using TanDEM-X satellite data. In this regard, optimization algorithm of Particle Swarm is used with the 4-D phase unwrapping of TanDEM-X satellite data. The study shows that the Particle Swarm Optimization algorithm is used to optimize the 4-D reconstruction of copper mineralization within 7 hours and post 2000 iterations with RMSE of 0.23 . The results show that the 4-D of copper mineralization improved morphological feature detection such as the depth of a copper mine and surrounding infrastructures. In conclusion, the integration of PSO with the 4-D phase unwrapping of TanDEM-X satellite data are an excellent promise approach for 4-D reconstruction of copper mineralization.
\end{abstract}

Volume 5 Issue 2 - 2019

\author{
Maged Marghany \\ Faculty Geospatial and Real Estate, Geomatika University \\ College, Malaysia
}

Correspondence: Maged Marghany, Faculty Geospatial and Real Estate, Geomatika University College, Kuala Lumpur, Malaysia,Email magedupm@hotmail.com

Received: December 21, 2018 | Published: March 18, 2019

Keywords: four-dimensional, copper mineralization, tandem-x, particle swarm optimization

\section{Introduction}

The TanDEM-X functions are based totally on (i) cross-track two SAR interferometry, (ii) along-track SAR interferometry and (iii) new SAR techniques. The three radar strategies evolve from the machine specification described with the aid of the TerraSAR-X satellite and the interferometric configuration itself. Owing to its manifold machine configurations, TanDEM-X is a bendy and multi-mode mission, which grants a broad variety of application possibilities. Dual Acrosstrack SAR interferometry is an identified method to decide the terrain topography. The usage of this technique is mounted for the calculation of phase variances calculated with two SAR antennas separated by a terrific baseline. This permits approximating the radar elevation angle to the phase centre of every photo decision cell, where the height facts are derived from the interferometric section alternate. ${ }^{1,2}$ Dual Alongtrack SAR interferometry is used to compute the velocities of shifting objects, which are a feature of a segment modification measurement whereby the two SAR antennas achieve complex SAR photographs of the identical location with a quick time lag. Therefore, new SAR strategies will establish the possibility of superior SAR systems that have yet now not or only incompletely been mounted on the ground or with aeroplanes.

The TanDEM-X operational consequence entails the synchronized operation of dual satellites hovering in contiguous configuration. The amendment compressions for the development are: (i) the orbits ascending nodes, (ii) the angle between the perigees, (iii) the orbital eccentricities and (iv) the phasing between the satellites. The main aim of the TanDEM-X mission is to create a unique third-dimensional image (3-D) of Earth, which is regular in superiority and excellence in precision. At present, the elevation models (DEMs) are offered free, which are of stumpy resolution, erratic or defective. Likewise, DEMs are regularly set up on numerous databases and surface survey techniques. In these regards, TanDEM-X, and TerraSAR-X additional for DEM quantity, which is premeditated to discontinue these disparities and supply a precise DEM, which have to authorize essential for numerous scientific and commercial demands.
DEMs provide a necessary footing for all topics in geological science, as a result the demand for particular and straightforward DEMs is of accurate prominence. DEMs, for instance, are a requirement for the improvement of geological maps. Supplementary, districts with volcanoes and predictable earthquake disaster require an extraordinarily up to date excessive resolution DEM to govern the deviations previous occasions. Moreover, straightforward and unique DEMs are required for the recognition of perilous developed zones being affected by way of failures., ${ }^{3,4}$ The international coverage of topographic data at an ample splendid three-dimensional resolution is at present now not reachable and would be delivered by using the TanDEM-X mission. The correct DEMs are necessary for geological, mining detections in spite of the negative aspects of synthetic aperture radar records due to speckles and object geometry distortions. Lopes et al., ${ }^{5}$ Touzi,${ }^{6} \mathrm{Yu} \&$ Scott, ${ }^{7}$ Hondt et al., ${ }^{8}$ Helmy \& El-Taweel, ${ }^{9}$ Marghany, ${ }^{10}$ Marghany ${ }^{10-13}$ Recently, Marghany ${ }^{14}$ developed a new technique for geological, mining detection in TerraSAR-X based Particle Swarm Optimization (PSO). In this view, Marghany ${ }^{14}$ and Marghany ${ }^{15}$ mentioned that Particle Swarm Optimization has accurate performance in solving several single and multi-objective optimization noises in TerraSAR-X data, such as noise despeckles, which approved by Riccardo et al., ${ }^{16}$ and Jin et al. ${ }^{17}$ This optimization of SAR speckles might produce high target visibility in coherence data.

This investigation has hypothesized that 4-D phase unwrapping through the use of Particle Swarm Optimization (PSO) can be used to reconstruct four-dimensional of copper mineralization from the TanDEM-X data. ${ }^{18}$ The foremost novelty of this work to use in the course of track interferometry of TanDEM-X information with the useful resource for setting up 4-D phase unwrapping primarily based absolutely Particle Swarm Optimization. The predominant intention of this work is to fortify the 4-D phase unwrapping algorithm principally based totally on Particle Swarm Optimization for 4-D copper mineralization reconstruction from TanDEM-X data.

\section{Algorithm}

Two algorithms are integrated to construct 4-D of the copper 
mineralization from TanDEM-X SAR data. These involve PSO and 4-D Phase unwrapping algorithms. The following sections explain this approach clearly. Succeeding Marghany, ${ }_{14}^{14}$ Particle Swarm Optimization (PSO) is a population-based randomly investigation process. It is assumed that there are $\mathrm{N}$ "particles" i.e., lineaments, faults, topographic breaks, bedding, depressions, lithologies, which are in SAR data.

Therefore, these geological aspects invasive contacts randomly appear in a "solution space". Thus the optimization hassle can be solved for information clustering; there is constantly a criterion (for example, the squared error function) for each and every single particle at their function in the solution space. The $\mathrm{N}$ particles will maintain transferring and calculating the criteria in each function, then rest, which is named as fitness in PSO pending the precise reaches the precise threshold. Hence, every geological feature (particle) two maintains two its coordinates in the solution space of TanDEM-X which are blended with the best fitness that has extraordinarily achieved with the aid of requested geological function i.e. particle. Indeed, the pixel of each feature i.e. particle $(m, n, l, t)$ denotes a probable solution to the optimization problem. Consistent with Kennedy \& Eberhart ${ }^{19}$ and Marghany ${ }^{14}$ each one moves the particle with a route and rate $v$

$$
p_{m, n, l, t}=v_{m, n, l, t}+p_{m, n, l, t}
$$

Here $p_{m, n, l, t}$ signifies particle and $v_{m, n, l, t}$ is the rate of the 4-D particle in the $i, j, k, t$ 4-D coordinates, respectively, which involving time-dimension. The mathematical model of the particle rate changing is given by:

$$
\begin{aligned}
& v_{m, n, l, t}=v_{m, n, l, t}+c_{2} r_{2}\left(\text { gbest }_{m, n, l, t}-p_{m, n, l, t}\right)+ \\
& +c_{1} r_{1}(\text { lbest } \\
& m, n, l, t \\
& \left.-p_{m, n, l, t}\right)
\end{aligned}
$$

Being, lbest $t_{m, n, l, t}$ is the local best particle, gbest $_{m, n, l, t}$ is the global best particle, $r_{1}$ and $r_{2}$ are random variables and $c_{1}, c_{2}$ are the swarm system variables. After each iteration the global best $g_{\text {best }}$ particle and the agent local best $l_{\text {best }}$ particle are evaluated based on the maximum fitness functions of all particles in the solution space. In this understanding, the quality map algorithm of 4-D phase unwrapping can be expressed mathematically based on equations 1 and 2 as follows:

$$
\begin{aligned}
v_{m, n, l, t} & =c_{1} \cdot r_{1}\left(p_{m, n, l, t}(t-1)\right. \\
& \left.-Q_{m, n, l, t}(t-1)\right)+c_{2} \cdot r_{2}\left(p_{m, n, l, t}(t-1)\right. \\
& \left.-Q_{m, n, l, t}(t-1)\right)+ \\
& w \cdot v_{m, n, l, t}(t-1) \\
Q_{m, n, l, t} & =v_{m, n, l, t}(t)+Q_{m, n, l, t}(t-1)
\end{aligned}
$$

Where $Q_{m, n, l, t}$ is the position of the particle for phase unwrapping based on the quality map, which is given by: $:^{14,15}$

$$
\begin{aligned}
& Q_{m, n, l, t}=\frac{1}{m \times n \times l x t} *\left(\left(\sum\left(\Delta \phi_{i, j, k, V}^{x}-\overline{\Delta \phi_{i, j, k, V}^{x}}\right)^{2}\right)^{0.5}+\left(\left(\sum\left(\Delta \phi_{i, j, k, V}^{y}-\overline{\Delta \phi_{i, j, k, V}^{y}}\right)^{2}\right)^{0.5}+\right.\right. \\
& \left(\left(\sum\left(\Delta \phi_{i, j, k, V}^{z}-\overline{\Delta \phi_{i, j, k, t}^{z}}\right)^{2}\right)^{0.5}+\left(\left(\sum\left(\Delta \phi_{i, j, k, V}^{t}-\overline{\Delta \phi_{i, j, k, t}^{t}}\right)^{2}\right)^{0.5},\right.\right.
\end{aligned}
$$

Where $\Delta \phi^{x}, \Delta \phi^{y}, \Delta \phi^{z}$, and $\Delta \phi^{t}$ are the unwrapped-phase gradients in the $x, y, z$, and $t$ directions, respectively? $\overline{\Delta \phi}^{x}, \overline{\Delta \phi}^{y}, \overline{\Delta \phi}^{z}$ and $\overline{\Delta \phi}^{t}$ are the mean of the unwrapped-phase gradient in $m \times n \times l x t$ a cube in $\Delta \phi^{x}, \Delta \phi^{y}, \Delta \phi^{z}$, and $\Delta \phi^{t}$, respectively. ${ }^{14,20}$ Further, $v_{m, n l, t}$ is the contemporary rate of the particles in $m \times n \times l x t$. The rate is structured by a set of rules that impact the dynamics of the swarm. Further then, there are several parameters must be considered such as the initial population, representation of position and velocity strategies, fitness function identification and the limitation. These parameters are for PSO performances. Subsequent Ibrahim et al., ${ }^{21}$ the initial swarm particles are initialized to contain 3000 facts of particles for $Q_{m, n, l, t}$ and rate $v_{m, n, l, t}$. The facts had been arbitrarily nominated in the azimuth and range directions in the TanDEM-X phase unwrapped results.

Following Kennedy \& Eberhart ${ }^{22}$ and El Meseery et al., ${ }^{23}$ Marghany, ${ }^{14}$ the error threshold is performed, the optimal solution is obtained post accomplishing a unique quantity of iterations or an accurate. $p_{i}$ is the non-public excellent function of the particle, $w$, $c$, are all constant factors, and $r$ is the random numbers uniformly distributed within the interval $[0,1]$. Thus the common swarm algorithm can be changed into a binary particle (Discrete Particle Swarm Algorithm DPSO) which handles particle values of either two or by means of above-given equations. On the word of El Meseery et al., ${ }^{23}$ the PSO can segment the geological features in SAR data. In this understanding, the input TanDEM-X data $T$ with $N$ points can be represented by set $T=\left\{x_{1}, x_{2} \ldots x_{N}\right\}$ where $x$ is the location of the 3 -D geological feature on the point $i$. The swarm algorithms consist of $M$ agents which are represented by the set $A=\left\{P_{i} \mid i=1,2 \cdots M\right\}$ where $p$ is a single solution particle from the solution space. Each particle decodes the problem using a binary array with the same length $N$ as the input SAR data. Consequently, the system denotes each particle $P_{i, j, k, t}$ by $P_{i}=\left\{p_{i j k} \mid k=1,2 \cdots N\right\}$ where $p_{i j k}$ has only two values a) 1 ( $\left.p_{i j k}=1\right)$; means that this point $(k)$ is a dominant point, or b) 0( $\left.p_{i j k}=0\right)$ which means that means this point $(k)$ is not a dominate point. ${ }^{23}$ The fitness is computed using the given equation:

$$
\max f i t n e s s\left(\mathrm{p}_{i, j, k, t}\right)=\left\{\begin{array}{lr}
-E / \varepsilon N & \text { if } E>\varepsilon, \\
D / \sum_{i=1}^{N} \sum_{j=1}^{N} \sum_{k=1}^{N} \sum_{t=1}^{N} P_{i, j, k, t} & \text { Otherwise }
\end{array}\right.
$$

Where $N$ is the number of pixels in the TanDEM-X satellite data, $D$ is the number of the solutions that were previously labelled as a possible dominant point $(P), E$ is the calculated error and $\varepsilon$ is the error threshold. On the word of El Meseery et al., ${ }^{23}$ and Marghany ${ }^{14}$ it should be perceived that when the inaccuracy is greater than the threshold $\varepsilon$, the fitness offers a negative value to lower the fitness value of the solution. On the contrary, the system favours the lower number of vertices. Consequently, Pesudo code of PSO is shown in Figure 1.

$$
\begin{aligned}
& \text { Equation }(4.1) \\
& \mathrm{v}[]=\mathrm{c} 0 * \mathrm{v}[] \\
& +\mathrm{c} 1 * \operatorname{rand}() * \text { (pbest[] - } \\
& +\mathrm{c} 2 * \text { rand }() * \text { (gbest[] - } \\
& \text { (in the original method, } \mathrm{c} 0 \\
& \text { researchers now play with } \\
& \text { Equation }(2) \\
& \text { present[] = present[] }+\mathrm{v}[] \\
& \text { For each particle } \\
& \text { Initialize particle } \\
& \text { END| }
\end{aligned}
$$$$
+\mathrm{c} 1 * \operatorname{rand}() *(\text { pbest[] - present[]) }
$$$$
+\mathrm{c} 2 * \operatorname{rand}() *(\text { gbest}[]-\text { present[] })
$$$$
\text { (in the original method, } \mathrm{c} 0=1 \text {, but many }
$$$$
\text { researchers now play with this parameter) }
$$

Figure I Pesudo code of PSO 


\section{Results and discussion}

At the beginning of the 20th century, largest mine of Chuquicamata, Chile was initiated by Guggenheim Brothers (Figure 2). This locates in the centre of the Atacama Desert near South America's west coast, which considers as the world's largest open-cast copper mine. The dominant feature of an oval structure- the largest-ever depression in the Earth's surface ever produced by human effort. ${ }^{14}$ Pair of Terra-X SAR satellite, which presents the TanDem-X mission that involves the high-resolution spotlight mode of $1 \mathrm{~m}$ resolution of $\mathrm{HH}$ polarization in X-band (Figure 3). Figure 3 shows the 400-metre deep mine, which is corresponding to $1876 \mathrm{~m}$ below the sea level. In addition, 3-D TanDEM-X data show clear infrastructures which are represented in water filtration tanks which can be observed clearly as square surfaces in the mine. It is located between $22^{\circ} 18^{\prime} 19.66^{\prime \prime} \mathrm{S}$ and $068^{\circ} 54^{\prime} 08.07^{\prime \prime} \mathrm{W}$ (Figure 4).

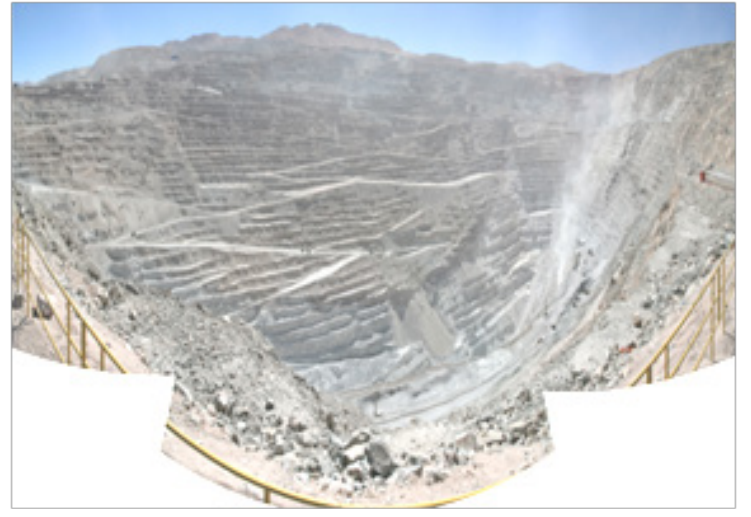

Figure 2 Largest mine of Chuquicamata.
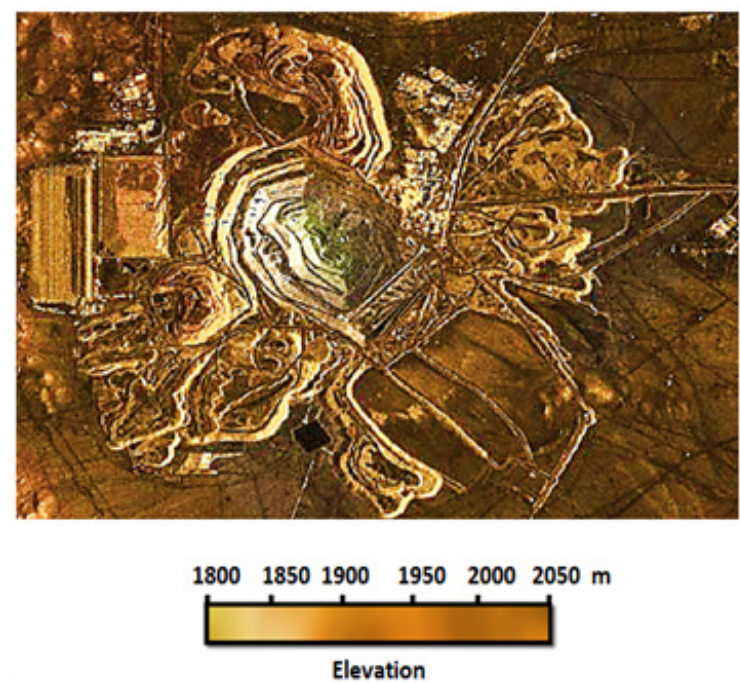

Figure 3 3-D copper mine from TanDEM-X data of high resolution spotlight mode.

Figure 5 spectacles the 4-D copper mineralization image from 3-D TanDEM-X data. It is recognizable that the perfect 4-D morphological feature detections from a diverse view angles. This incorporates subterranean of copper mine within $400 \mathrm{~m}$ depth among contiguous mountains. Moreover, cavernous portrayals of the superiority of the substructures. The geomorphology of copper mineralization being to be more obvious with rotation angle of $180^{\circ}$ (Figure 5b). The involving of 4-D dimension intensifications the deeper conception of the scene as diverse landscapes, which are experiential with altered view angles from $0^{\circ}$ to $360^{\circ}$. The bright color along the object edges is corresponding to the fourth-dimension, which represents time of 5 days. This agrees with the work of Ibrahim et al., ${ }^{21}$ and Marghany. ${ }^{14}$

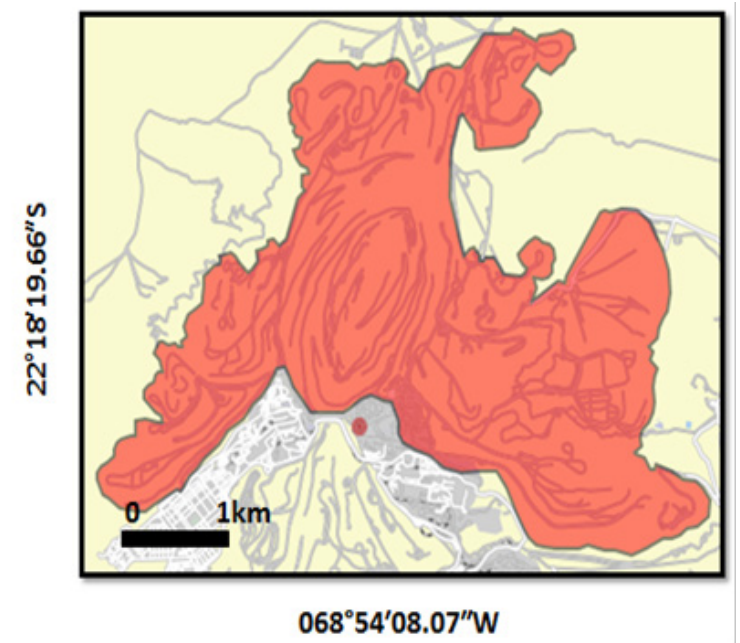

Figure 4 Geographical location of Chuquicamata, Chile.
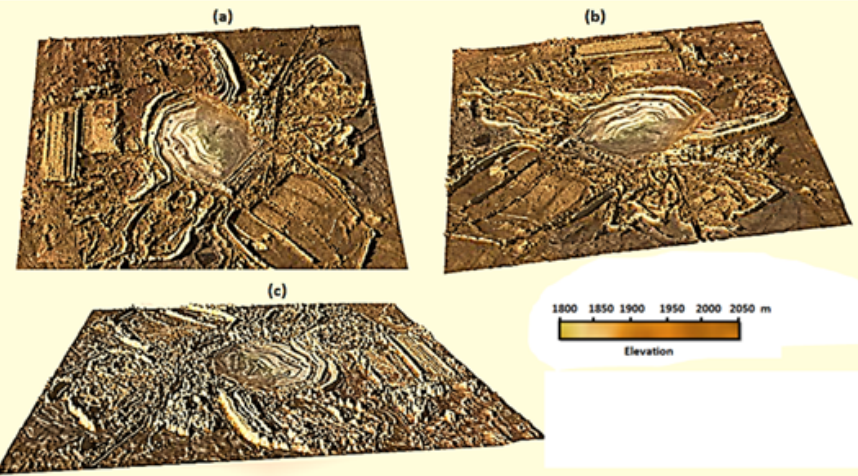

Figure 5 4-D copper mineralization from different view angles (a) $0^{\circ}$, (b) $180^{\circ}$ and (c) $360^{\circ}$.

The implementation of PSO with 4-D phase unwrapping assisted to determine most fulfilling grows regions across the persevering with unwrapping of edges. In this view, PSO synchronized the voxels on each aspect of the area (Figure 4) In addition, 4-D phase unwrapped algorithms constructed the discontinuity in quality order. This is fabulous in the excessive depth line or curve of fixed length and locally low curvature boundary is recognized to exist between edge factors and excessive noise ranges in TanDEM-X data. PSO, conversely, optimizes the gaps stays between discontinuity edges. In this regard, 4-D phase unwrapping based PSO is a choicest search for actual partial values, which are present at the boundary of copper mineralization and the optimization of 4-D section unwrapping in hypercube can reconstruct the 3-D object displacement with extra 4th dimension. Finally, 4-D phase unwrapping based totally PSO approves for the dependable unwrapping of low signal to noise ratio (SNR). This finding out about ought to enhance of 3-D phase unwrapping proposed by means of Peer et al., ${ }^{24}$ Hussien et al. ${ }^{25}$ Karout, ${ }^{26}$ and Marghany. ${ }^{14,27}$

\section{Conclusion}

This investigation has established a new method for geological copper mining detection. In this regard, optimization algorithm of Particle Swarm is used with the 4-D phase unwrapping of 
TanDEM-X satellite data. The finding out about is suggesting that the Particle Swarm Optimization algorithm is used to optimize the 4-D reconstruction of copper mineralization within 7hours and put up 2000 iterations with RMSE of 0.23 . The consequences exhibit that the 4-D of copper mineralization extended morphological characteristic detection such as the depth of a copper mine and surrounding infrastructures. It can be stated that the integration of PSO with the 4-D phase unwrapping of TanDEM-X satellite data are a high-quality promise method for 4-D reconstruction of copper mineralization.

\section{Acknowledgments}

None.

\section{Conflicts of interest}

Author declares there are no conflicts of interest.

\section{References}

1. Marghany M. Three-Dimensional Coastal Geomorphology Deformation Modelling Using Differential Synthetic Aperture Interferometry. Zeitschrift fur Naturforschung A-Journal of Physical Sciences. 2012;67(6):419.

2. Marghany M. Hybrid Genetic Algorithm of Interferometric Synthetic Aperture Radar for Three-Dimensional Coastal Deformation. InSoMeT. 2014. p. 116-131.

3. Marghany M. DEM reconstruction of coastal geomorphology from DINSAR. International Conference on Computational Science and Its Applications. 2012. p. 435-446.

4. Marghany M. DInSAR technique for three-dimensional coastal spit simulation from radarsat-1 fine mode data. Acta Geophysica. 2013;61(2):478-493.

5. Lopes A, Touzi R, Nezry E. Adaptive speckle filters and scene Heterogeneity. IEEE. 1990;28(6):992-1000.

6. Touzi R. A review of speckle filtering in the context of estimation theory. IEEE Transactions on Geosciences and Remote Sensing. 2002;40(11):2392-2404.

7. YuY,ScottTA. Speckle reducing anisotropic diffusion.IEEETransactions on Geoscience and Remote Sensing. 2002;11(11):1260-1270.

8. Hondt OD, Ferro-Famil L, Pottier E. Nonstationary spatial texture estimation applied $\mathrm{t}$ adaptive speckle reduction of SAR data IEEE Transactions on Geosciences and Remote Sensing Letter. 2006;3(4):476-480.

9. Helmy AK, El-Taweel GS. Speckle Suppression of Radar Images Using Normalized Convolution. Journal of Computer Science. 2010;6(10):1125-1129.

10. Marghany M. 3-D coastal bathymetry simulation from airborne TOPSAR polarized data. Bathymetry and Its Applications. 2012. p. $57-76$.
11. Marghany M. Three-Dimensional Lineament Visualization Using Fuzzy B-Spline Algorithm from Multispectral Satellite Data. 2012. p. 213-232.

12. Marghany. Particle Swarm Optimization for Geological Feature Detection from PALSAR Data. 35th Asian Conference of remote sensing, at Nay Pyi Taw. 2014.

13. Marghany M. Multi-Objective Evolutionary Algorithm for Oil Spill Detection from COSMO-SkeyMed Satellite. ICCSA. 2014. p. 355-371.

14. Marghany 2015. Copper mine automatic detection from TerraSAR-X using particle swarm optimization. CD of 36th Asian Conference on Remote Sensing. 2015.

15. Marghany M. Advanced Remote Sensing Technology for Tsunami Modelling and Forecasting. USA: Taylor \&Francis Group, CRC Press; $2018.302 \mathrm{p}$.

16. Riccardo P, Kennedy J, Blackwell T. Particle swarm optimization-An overview. Swarm Intell. 2007;1(1):33-57.

17. Jin Yisu, Joshua Knowles, Lu Hongmei, et al. The landscape adaptive particle swarm optimizer. Applied Soft Computing. 2008;8:295-304.

18. Ali RY. A new hybrid particle swarm optimization approach for structural design optimization in the automotive industry. Proceedings of the Institution of Mechanical Engineers, Part D: Journal of Automobile Engineering. 2012;226(10):1340-1351.

19. Kennedy J, Eberhart R. Particle Swarm Optimization. 1995. p. 1942-1948.

20. Schwarz O. Hybrid phase unwrapping in laser speckle interferometry with overlapping windows. Shaker Verlag; 2004.

21. Ibrahim S, Abdul Khalid NE, Manaf M. Computer aided system for brain abnormalities segmentation. Malaysian Journal of Computing. 2010;1(1):22-39.

22. Kennedy James, Russell C Eberhart. A discrete binary version of the particle swarm algorithm. IEEE International Conference. 1997.

23. El Meseery M, El Din MF, Mashali S, et al. Sketch recognition using particle swarm algorithm. 16th IEEE International Conference on Image Processing. 2009;7-10.

24. Peer ES, Van Den Bergh F, Engelbrecht AP. Using neighbourhoods with the guaranteed convergence PSO. Proceedings of the 2003 IEEE. 2003. p. 235-242.

25. Hussein SA, Gdeist M, Burton D, et al. Fast three-dimensional phase unwrapping algorithm based on sorting by reliability following a noncontinuous path. Proc SPIE. 2005.

26. Karout S. Two-Dimensional Phase Unwrapping. Ph.D. Theses Liverpool John Moores University; 2007.

27. Lee JS, Schuler D, Ainsworth TL, et al. On the estimation of radar polarization orientation shifts induced by terrain slopes. IEEE Transactions on Geosciences and Remote Sensing. 2002;40(1):30-41. 